\title{
Ein Hörgerät könnte Legasthenikern helfen
}

\begin{abstract}
Leiden Kinder an einer Lese-Rechtschreib-Schwäche, sind oft Probleme mit der Verarbeitung von Sprache die Ursache: Das Hörzentrum wandelt wichtige Laute weniger effektiv um als bei Kindern ohne die Störung. Dadurch fällt es Legasthenikern unter anderem schwer, ähnlich klingende Wörter und Laute zu unterscheiden, wie beispielsweise "Katze" und "Tatze". Sie lernen dadurch langsamer, Laute mit bestimmten Buchstaben zu verbinden. Zudem ist es für Legastheniker schwieriger, sich auch bei Störgeräuschen auf eine Stimme zu konzentrieren.
\end{abstract}

- Wissenschaftler von der Northwestern University in Evanston (Illinois, USA) wollten wissen, ob speziell für den Schulunterricht konzipierte Hörhilfen legasthenischen Kindern helfen können. Die Forscher statteten deshalb 38 legasthenische Kinder zwischen acht und 14
Jahren mit Hörhilfen aus. Ein Jahr lang trugen die Schüler im Unterricht einen kleinen Empfänger am Ohr, der die Stimme des mit einem Mikrofon ausgerüsteten Lehrers verstärkte.

Dank des Geräts konnten die Schüler den Lehrer auch

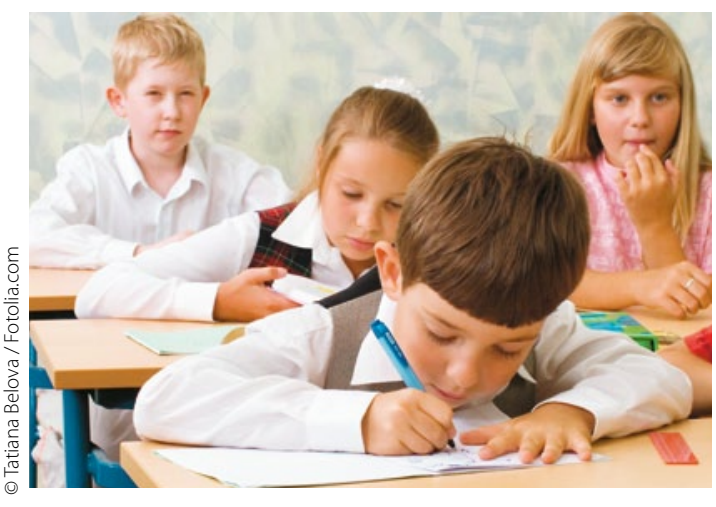

Beruht Dyslexie auf Hörfehlern? dann deutlich hören, wenn es viele ablenkende Störgeräusche gab. Bei den Kindern, die die Hörhilfen getragen hatten, reagierte das Hörzentrum konsistenter und genauer als noch ein Jahr zuvor. Auch konnten diese Kinder deutlich besser lesen als die Teilnehmer ohne Hörhilfe.

K. MALBERG =
- J. Hornickel et al.
(Korrespondenzadresse: Nina Kraus: Audito- ry Neuroscience Laboratory, Northwestern University, Evanston, II, 60208, USA; E-mail: nkraus@northwestern.edu.) Assistive listening devices drive neuroplasticity in children with dyslexia. published online before print 2012, September 4 doi: 10.1073/pnas1206628109

\section{Kommentar}

Alle Teilnehmer besuchten private Schulen, die speziell auf Kinder mit Lese-RechtschreibSchwäche ausgerichtet sind. Zu Studienbeginn hatten die Forscher die Lesefähigkeit der Kinder und die Aktivität in dem im Hirnstamm gelegenen Teil ihres Hörzentrums gemessen. Wie erwartet erzeugte das Hörzentrum vor allem bei Konsonanten häufig falsche oder ungenaue Signale. Grundsätzlich war die Hörfähigkeit der Kinder aber völlig normal.

\section{Inapparentes Vorhofflimmern häufig}

\section{Der Schrittmacher bringt es an den Tag: Klinisch inapparentes Vorhof- flimmern ist häufig und geht mit einem erhöhten Schlaganfallrisiko einher.}

- Die Software moderner Schrittmacher erlaubt es, auch nur ganz selten und kurz dauernd auftretende Episoden mit hoher Vorhoffrequenz über lange Zeiträume hinweg aufzuzeichnen und $\mathrm{zu}$ analysieren. Dies erfolgte nun bei 2580 Patienten über 65 Jahre mit Hypertonie und ohne bekanntes klinisch manifestes Vorhofflimmern, die einen Schrittmacher neu implantiert bekommen hatten. Die Vorhoffrequenz wurde über einen Zeitraum von drei Monaten hinweg überwacht und die Patienten anschließend zweieinhalb Jahre lang weiter beobachtet.
In den drei Monaten nach der Schrittmacherimplantation stellte man bei 261 Patienten $(10,1 \%)$ klinisch nicht erkanntes tachykardes Vorhofflimmern fest.

Dieses Phänomen ging mit einem 5,6-fach erhöhten Risiko für ein klinisch manifestes Vorhofflimmern und mit einem 2,5-fach erhöhten Risiko für einen Apoplex oder eine systemische Embolie einher.

\section{- J. S. Healey et al.}

(Korres.: Dr. Conolly connostu@phri.ca): Subclinical atrial fibrillation and the risk of stroke. New Engl. J. Med. 2012; 366: 120-129.

\section{Kommentar}

Die Untersuchung bringt eine schmerzliche Tatsache zu Tage, die wir schon immer geahnt haben, die wir aber mit den relativ grobmaschigen Methoden des üblichen 24-h-Langzeit-EKG nicht erfassen konnten. Allerdings ist unter den gegenwärtigen Bedingungen noch nicht klar, welche Konsequenzen für die Apoplexprophylaxe aus der Untersuchung gezogen werden sollten. Die Autoren konstatieren zwar, dass auch die
Patienten mit inapparentem Vorhofflimmern bei einem CHADS2-Score $>2$ ein jährliches Risiko für einen Apoplex von 4\% hatten und damit die Indikation für eine Antikoagulation bestünde. Warum aber sollte man überhaupt den Score berechnen, wenn unter den Bedingungen der heute üblichen Diagnostik kein Vorhofflimmern erfasst wird?

H. S. FÜESSL — 